\title{
Improved Detection of Cervical Cancer and High Grade Neoplastic Lesions by a Combination of Conventional Cytology and DNA Automated Image Cytometer
}

\author{
Zuming Li ${ }^{1}$, Min Zhang ${ }^{2}$, Hua $\mathrm{Li}^{2}$ \\ ${ }^{1}$ Yiling Women and Children Hospital, Yichang, Hubei, China; ${ }^{2}$ Zhongshan Hospital, Wuhan, Hubei, China. \\ Email: xrsun2002@yahoo.com
}

Received May $12^{\text {th }}, 2010$; revised June $4^{\text {th }}, 2010$; accepted June $11^{\text {th }}, 2010$.

\begin{abstract}
OBJECTIVE: To reduce false-negative rates of population based cervical screening programs employing conventional cytology in combination with automated DNA image cytometer. METHODS: Involved cervical samples from a total of 3603 women were taken by a cervix brush and then placed into a fixative solution. The cells were separated from mucus by mechanical and chemical treatment after which they were deposited onto microscope slides by a cytospin. Two slides were prepared from each case; one slide was stained by Papanicolaou stain for conventional cytology examination, while the other slide was stained by a DNA specific and stoichiometric stain. The latter slide was used to determine the relative amount of DNA in the cell nuclei in order to assess the ploidy status of the epithelial cells. Enrolled in the study, 157 women were followed by colposcopy examination where punch biopsies were taken from the visible lesions or from suspicious areas. The results of the conventional cytology were then compared to the DNA image cytometer for all samples. RESULTS: Histopathology diagnosed 51 lesions from the 132 biopsied cases as CIN2 or higher, including 27 CIN2, 16 CIN3 and 8 invasive cancers. Conventional cytology correctly identified 29 of the 51 high grade CIN and invasive cancer, while DNA image cytometer correctly identified 38 high grade CIN and invasive cancer using the criterion that at least three cells were found on the slide that contained DNA amount in excess of $5 c$. 42 out of 51 high grade CIN and invasive cancer were found by conventional cytology in combination with DNA image cytometer. Sensitivities were $56.8 \%, 74.5 \%$ and $82.4 \%$, while specificities were $86.2 \%, 81.5 \%$ and $81.5 \%$ in conventional cytology, DNA image cytometer and combination both cytology and DNA image cytometer respectively. CONCLUSION: The study demonstrated that screening for high grade neoplastic lesions and cervical cancer by DNA image cytometer or combination of conventional cytology and DNA image cytometer is more sensitive than conventional screening approach.
\end{abstract}

Keywords: Cervical Intraepithelial Neoplasia (CIN), Conventional Cytology, Image Cytometry, Ploidy, Aneuploid Cells, Invasive Cervical Cancer

\section{Introduction}

The cervico-vaginal cytologic test is a powerful tool for detecting cancerous and precancerous (neoplastic) cervical lesions. In countries where well organized population based screening programs were implemented, the incidence of invasive cervical cancers and mortality due to cervical cancer have dropped dramatically [1-3]. However, there are many countries in the world that population based screening has not yet been performed due to the relative high cost and lack of skilled technologists.

The correct diagnosis of high grade cervical intraepi- thelial neoplasia and invasive cancer is a difficult task in the cytological evaluation of cervico-vaginal smears [4-6]. The performance of several laboratories was summarized by van der Graaf et al. [7] who shown that only about half of biopsy-documented invasive cancers were appropriately recognized by conventional cytology of cervical smears. To increase the diagnostic accuracy and to avoid mistakes by cytopathology, several cytometry based systems have been developed over the past three decades for the detection of abnormal DNA content of the cervical epithelial cells [8-11].

The study related to the investigation to determine 
conventional cytology in combination with ploidy status assessment of epithelial cervical cells by a fully automated image cytometer could be used for an effective detection of high grade of cervical intraepithelial neoplasia and invasive cervical cancer.

\section{Materials and Methods}

\subsection{Sample Collection and Sample Preparation}

The study involved a total of 3,603 women from the Yiling region (Hubei Province, China) and included 1/3 women undergoing their routine screening, as well as $2 / 3$ women undergoing their first screening test. The women were invited to come to either Yiling Women and Children Hospital or to one of the several local hospitals. The samples were obtained by employing a cervix brush. After sample was taken, the brush tip was immediately immersed in $20 \mathrm{ml}$ of fixative (Landing Medical High-Tech Company, Wuhan, China) in $30 \mathrm{ml}$ plastic vials. All vials from the collection sites were further processed by first adding Dithiothreitol (DTT, Sigma) to the final concentration of $0.1 \%$ of DTT. The cell suspensions were then mildly agitated for 1 hour to release cells from the brush into the suspension and to disaggregate the cells from mucus and cell clusters. The cells were then washed twice with $50 \%$ alcohol. Two slides were then prepared from each specimen by cytospinning the cells onto microscope slides forming a uniform monolayer. The cytospin preparation contained on average 10,000 cervical cells deposited in the middle of the slide. One of the two slides was stained by the Papanicolaou stain for conventional cytology reading of the slide, and the other slide was stained with the DNA specific and stoichiometric (Feulgen-Thionin) stain (12) for the ploidy status assessment of the cells on the slide by image cytometry method.

Women presenting any cytological abnormalities were directed to repeated cytology and for colposcopy examination. Of over 157 invited women, 132 women came for colposcopy examination and punch biopsies were taken from the visible lesions or from suspicious areas.

\subsection{Cytology}

All Papanicolaou stained slides were examined by two cytopathologists. The smears were classified into one of the five groups according to the Bethesda system: 1) within normal limits; 2) with ASCUS (atypical squamous cells of undermined significance); 3) low-grade squamous intraepithelial lesion (LSIL); 4) high-grade squamous intraepithelial lesion (HSIL); and 5) squamous cell carcinoma.

\subsection{Image Cytometer}

All Thionin-Feulgen stained slides were scanned by the
Lanidng DNA image cytometer. The images of the cell nuclei were projected onto the CCD that was positioned in the primary image plane of the 20 times objective, resulting in an effective pixel size of $0.34 \mathrm{um} \times 0.34 \mathrm{um}$ $\left(\sim 0.1 \mathrm{um}^{2}\right)$. A typical image of the nucleus of a cervical epithelial cell is represented with 700-900 picture elements (pixels). The image of each cell nucleus was captured in the exact focus and the nuclear material was segmented from the background. For each nucleus, 99 nuclear features were calculated including morphological features, photometric features, discrete texture features, Markovian and non-Markovian texture features, runlength features and fractal features. These features were then used to identify objects as true cells or "junk" (overlapping cell nuclei, out of focus cell nuclei, cellular debris) as well as to classify the nuclei to belong to different cell types. The system was trained to perform this task in a fully automated way. The mean integrated optical density (IOD) value of the $2 \mathrm{c}$ diploid cells from each slide was used to normalize the optical density features to eliminate any stain intensity variations within the slides.

On average, from each slide approximately 5,000 quality images of cell nuclei were collected and stored in the computer memory of the cytometer. The nuclei, determined by the system to belong to the epithelial cells, were used to calculate and plot the DNA distribution histograms. The histograms were called normal if corresponded to diploid cells with a low proliferation fraction $\left(\mathrm{S}+\mathrm{G}_{2}+\mathrm{M}\right)$ according to the classification of Auer et al. [13]. All other histograms suggesting the presence of: 1) any cells with DNA $>5 \mathrm{c}$; or 2) diploid cells with a very high proliferation rate where $10 \%$ or more of the total cells were found in the proliferation fraction; or 3) a population of aneuploid stem cells, were called potentially abnormal and the women corresponding to any of abnormal histogram were called for a colposcopy examination. All women, correlated with normal histograms were not further examined unless any atypia was found by conventional cytology.

\subsection{Pathology}

132 biopsy specimens were taken from suspicious areas for histo-pathological diagnosis. Pathology report of each specimen was generated independently by two experienced pathologists.

\subsection{Statistical Analysis}

Sensitivity and specificity are calculated using the following formula:

$$
\begin{gathered}
\text { Sensitivity }=\frac{\text { True Positive }}{\text { True Positive }+ \text { False Positive }} \times 100 \% \\
\text { Specificity }=\frac{\text { True Negative }}{\text { True Negative }+ \text { False Negative }} \times 100 \%
\end{gathered}
$$




\section{Results}

\subsection{General Results}

Of the total of 3.603 smears, conventional cytology found 3,368 smears were within normal limit $(93.5 \%)$, and 233 had some form of cytological atypia $(6.5 \%)$ including 180 with ASCUS (5\%), 36 with LSIL (1\%), 19 with HSIL $(0.5 \%)$. Of these only 157 women were available for follow up by histopathology. Of the 3,206 cases 3,603 (91.1\%) DNA histograms were considered normal and 397 (8.9\%) cases were judged suspicious using the above criteria. Of these, 329 had 1-2 cells with DNA amount greater than $5 \mathrm{c}$ and the rest were found with 3 or more cells with DNA amount greater than $5 \mathrm{c}$ or aneuploid stem cells (Table 1).

\subsection{Comparison of the Results of Conventional Cytopathology with DNA Image Cytometer}

Table 2 shows the results of the 132 women that were examined by colposcopy and had taken punch biopsies. Pathology diagnosis by two histopathlogists was used as the 'truth' for sensitivity and specificity (false negative and false positive rate) calculations. There were 51 cases of $\mathrm{CIN} 2 / \mathrm{CIN} 3 / \mathrm{CIS} / \mathrm{Ca}$ lesions and 81 cases of Nor$\mathrm{mal} / \mathrm{benign} / \mathrm{CIN} 1$ in total of 132 cases. There were 8 cases of invasive cancers, 16 cases of CIN3/CIS and 27 cases of CIN2 lesions diagnosed by histopathlogists for the total of 51 lesions of $\mathrm{CIN} 2 / \mathrm{CIN} 3 / \mathrm{CIS} / \mathrm{Ca}$ type. If conventional cytology were to be used to refer all cases of LSIL/HSIL/Ca to colposcope and biopsy, 29 cases (including 4 LSIL without any $>5 \mathrm{c}$ aneuploid cells) were corrected identified and 22 from the total 51 cases were missed.

For DNA image cytometer, using the criterion that 3 or more cells with the DNA amount exceeding $5 \mathrm{c}$ is sufficient to call the sample suspicious to be examined by cytologist, this approach correctly identified 38 of 51 CIN2/CIN3/CIS/Ca lesions. Conventional cytolgy in combination with DNA image cytometer, 42 of 51 CIN2/ $\mathrm{CIN} 3 / \mathrm{CIS} / \mathrm{Ca}$ lesions were correctly identified using the criterion that 3 or more cells with the DNA amount exceeding $5 \mathrm{c}$ or LSIL or HSIL without 3 or more $>5 \mathrm{c}$ cells.

From the data presented in Table 2, sensitivity and specificity of conventional cytology, DNA image cytometer and conventional cytology combined with DNA image cytometer were calculated for high grade lesions of CIN2, CIN3/CIS and invasive cancer. Sensitivity of conventional cytology was $56.8 \%$ at $86.4 \%$ specificity. The criterion of at least 3 cells containing DNA amount greater than $5 \mathrm{c}$ would give sensitivity and specificity values of $74.5 \%$ and $81.5 \%$, respectively, while for the cytology combined DNA image cytometer were $82.4 \%$ and $81.5 \%$, respectively.

Table 1. The results of TBS diagnosis and DNA ploidy analysis in 3603 cervical samples

\begin{tabular}{|c|c|c|c|c|c|c|}
\hline \multirow{2}{*}{\multicolumn{2}{|c|}{ DNA ploidy Analysis }} & \multicolumn{5}{|c|}{ Cytology } \\
\hline & & Normal & ASCUS & LSIL & HSIL & Total \\
\hline & Normal & 3112 & 90 & 4 & 0 & 3206 \\
\hline $1-2$ & $>5 \mathrm{c}$ aneuploid & 244 & 75 & 8 & 2 & 329 \\
\hline & $>5 \mathrm{c}$ aneuploid & 12 & 15 & 24 & 17 & 68 \\
\hline & Total & 3368 & 180 & 36 & 19 & 3603 \\
\hline
\end{tabular}

Table 2. Comparative results of histopathology, conventional cytology and DNA image cytometer of 132 biopsy cases

\begin{tabular}{|c|c|c|c|c|c|c|c|}
\hline \multirow{2}{*}{ Pathology } & \multicolumn{4}{|c|}{ Conventional Cytology } & \multicolumn{3}{|c|}{$\begin{array}{c}\text { DNA Image Cytometer } \\
\text { Positive (aneuploidy }>5 \mathrm{c} \text { ) }\end{array}$} \\
\hline & Normal & ASCUS & LSIL & HSIL & Negative & $1-2$ cells & 3 or more cells \\
\hline Norma/benign (47) & 2 & 42 & 3 & 0 & 2 & 39 & 6 \\
\hline CIN1 (34) & 3 & 23 & 5 & 3 & 2 & 23 & 9 \\
\hline CIN2 (27) & 4 & 10 & 11 & 2 & 1 & 9 & 17 \\
\hline CIN3 or CIS (16) & 0 & 5 & 6 & 5 & 1 & 2 & 13 \\
\hline Invasive cancer $(8)$ & 0 & 3 & 3 & 2 & 0 & 0 & 8 \\
\hline
\end{tabular}




\section{Discussion}

Similarly to other studies [14,15], DNA image cytometer could increase sensitive in detecting high grade CIN lesions and cervical cancer. In this study, histopathology diagnosed 51 lesions from the 132 biopsied cases as CIN2/CIN3 or higher, of which there were 8 invasive cancers. Conventional cytology correctly identified 5 of the 8 invasive cancers while DNA assisted cytology correctly identified 8 invasive cancers using the criterion that at least three cells were found on the slide that contained DNA amount in excess of 5c. DNA image cytometer correctly identified 30 cases out of 43 CIN2/ CIN3/CIS lesions versus only 23 by conventional cytology. The results of this study suggest that screening for cervical cancer by DNA image cytometer is more sensitive than conventional cytology in detecting high grade CIN lesions and cervical cancer although at the expense of a slightly reduced specificity $(86.4 \% v s .81 .5 \%)$. Cytology combined with DNA image cytometer was also raised more sensitive than DNA image cytometer only.

It could be argued that in this study the conventional cytology was not at the level of a typical cytology laboratory. Kok et al. [5] shown that only 23 cases from 71 ( $\sim 33 \%$ sensitivity) invasive cancer patients were suspected as carcinoma by cytology, the results comparable to those reported in this study. The performance of several laboratories was summarized by van der Graaf et al. [7] who reported that only about half of biopsy-documented invasive cancers were appropriately recognized by cytology of cervical smears.

In this work, cells with DNA amount greater than $5 \mathrm{c}$ were used as the sole indicator of potential presence of cancer [13]. There is a large body of literature that suggested that cervical cancer and high grade lesions are associated with aneuploid population of cells [16]. These are readily detected if sufficient number of cells could be measured and when implemented and tested could improve the performance of this approach.

Cytology combined with DNA image cytometer could increase sensitive in detecting high grade CIN lesions and cervical cancer compared to conventional cytology. It is also suggested DNA image cytometer can be used to cervical cancer screening programs.

\section{Acknowledgements}

The authors would like to thank Dr. Xiao Rong Sun and Dr. Jian Wang for their assistance.

\section{REFERENCES}

[1] S. Liu, R. Semenciw, A. Probert and Y. Mao, "Cervical Cancer in Canada: Changing Patterns in Incidence and Mortality," International Journal of Gynecological Cancer, Vol. 11, 2001, pp. 24-31.

[2] G. H. Anderson, D. A. Boyes, J. L. Benedet, J. C.
LeRiche, J. P. Matisic, K. C. Suen, A. J. Worth, A. Millner and O. M. Bennett, "Organisation and Results of the Cervical Cytology Screening Programme in British Columbia, 1955-1985," Britain Medical Journal, Vol. 296, 1988, pp. 975-978.

[3] F. Guidozzi, "Screening for Cervical Cancer," Obstetrical \& Gynecological Survey, Vol. 51, 1996, pp. 247-252.

[4] J. D. Gay, L. D. Donaldson and J. R. Goellner, "Falsenegative Results in Cervical Cytologic Studies," Acta Cytologica, Vol. 29, 1985, pp. 1043-1046.

[5] M. R. Kok, M. E. Boon, P. G. Schreiner-Kok, L. G. Koss, "Cytological Recognition of Invasive Squamous Cancer of the Uterine Cervix: Comparison of Conventional Light-Micro-Scopical Screening and Neural NetworkBased Screening," Human Pathology, Vol. 31, 2000, pp. 23-28.

[6] S. E. Barton, D. Jenkins, A. Hollingworth, J. Cuzick and A. Singer, "An Explanation for the Problem of FalseNegative Cervical Smears," British Journal of Obstet Gynaecol, Vol. 96, 1989, pp. 482-485.

[7] Y. van der Graaf, G. P. Vooijs, H. L. J. Gaillard and D. M. Go, "Screening Errors in Cervical Cytologic Screening," Acta Cytologica, Vol. 31, 1987, pp. 434-438.

[8] D. M. Garner, A. Harrison and C. F. MacAulay, "CytoSavantTM and its Use in Automated Screening of Cervical Smears. Compendium on the Computerized Cytology and Histology Laboratory,” In: G. L. Wied Ed., Tutorial of Cytology, Chicago, 1994, pp. 346-352.

[9] M. Bibbo, P. H. Bartels, H. E. Dytch and G. L. Wied, "Cell Image Analysis. Comprehensive Cytopatholgy," In: M. I. Bibbo Ed., W. B. Saunders Company, Philadelphia, 1991, pp. 965-983.

[10] A. Bocking and V. Q. Nguyen, "Diagnostic and Prognostic Use of DNA Image Cytometry in Cervical Squamous Intraepithelial Lesions and Invasive Carcinoma," Cancer, Vol. 102, 2004, pp. 41-54.

[11] A. Mayer, M. Hockel, O. Thews, K. Schlenger and P. Vaupel, "Impact of Oxygenation Status and Patient Age on DNA Content in Cancers of the Uterine Cervix," International Journal of Radiation Oncology Biology Phys$i c s$, Vol. 56, 2003, pp. 929-936.

[12] X. R. Sun, J. Wang and L. Li, "Methods of DNA Quantitative Cytology. DNA Quantitative Cytology,” In: X. R. Sun and J. Wang Eds., Hubei Science and Technology press, Wuhan, 2006, pp. 22-26.

[13] G. Auer, T. O. Caspersson and A. S. Wallgren, "DNA Content and Survical in Mammary Carcinoma," Analytical \& Quantitative Cytology, Vol. 2, 1980, pp. 161-165.

[14] M. Guillaud, L. B. John, B. C. Scott, et al., "DNA Ploidy Compared with Human Papilloma Virus Testing (Hybrid Capture II) and Conventional Cervical Cytology as a Primary Screening Test for Cervical High-Grade Lesions and Cancer in 1555 Patients with Biopsy Confirmation," Cancer, Vol. 107, 2006, pp. 309-318.

[15] H. Tong, R. Shen, Z. M. Wang, et al., "DNA Ploidy Cytometry Testing for Cervical Cancer Screening in China 
(DNA CIC Trial): A Prospective Randomized, Controlled Trial," Clinical Cancer Research, Vol. 15, 2009, pp. 6438-6445.

[16] A. Bocking and V. Q. H. Nguyen, "Diagnostic and Prog- nostic Use of DNA Image Cytometry in Cervical Squamous Intraepithelial Lesions and Invasive Carcinoma," Cancer Cytopathology, Vol. 102, 2004, pp. 41-54. 